\title{
92. The Relative Abundance of the Chemical Elements in White Dwarf and its Electrification.
}

By Seitarô Suzuki.

Kyushu Imperial University, Fukuoka.

(Comm. by T. Terada, M.I.A., Oct. 12, 1931.)

The recent advancement of astronomy both observational and theoretical has thrown a new light on the property of white dwarfs. Their internal constitution is however obscure especially as regards the electrical state and the matter contained in them. To elucidate these points, it is necessary to investigate the dissociative equilibrium of various elements in the star. For this we make first the following assumptions.

(i) The interior of the white dwarf is in the degenerate state of perfect gases in the sense of the statistics of Fermi and Dirac.

(ii) All atomic nuclei of various elements are stripped of the extranuclear electrons (valency electrons).

(iii) All elements are formed of protons and electrons, in a definite proportion.

(i) was enunciated first by Fowler $^{1)}$ and subsequently adopted by Stoner, Anderson and Milne..) (ii) is the direct outcome from the immense hotness of the star interior. (iii) is regarded as Prout's theory.

Let $E, P, e, U$ represent respectively a nucleus, a proton, an electron, and the heat of formation.

$$
\mu P+\nu e \rightleftarrows E+U
$$

is a "chemical equation" of nuclear change, $\mu, \nu$ being positive integers. They are obtained approximately by

$$
\mu=\frac{m_{R}}{m_{P}}, \quad \nu=\mu-Z_{N},
$$

$Z_{E}$ being the atomic number.

Denote the mass of a particle, its statistical weight, the concentration by $m, q, c$, the suffix referring to the respective element. The equation of the thermal equilibrium among nuclei, dissociated protons and

1) R. H. Fowler: Monthly Not., 87 (1926), 114.

2) E. C. Stoner: Phil. Mag., 7 (1929), 63 ; W. Anderson: ZS. f. Phys. 66 (1930), 280 ; E. A. Milne: Monthly Not., 91 (1930), 4. 
electrons when all of them are degenerate is calculated easily from Milne's formulae."

$$
\frac{1}{m_{E}}\left(\frac{c_{E}}{q_{F}}\right)^{\frac{3}{3}}=\frac{\mu}{m_{P}}\left(\frac{c_{P}}{q_{P}}\right)^{\frac{2}{3}}+\frac{\nu}{m_{e}}\left(\frac{c_{e}}{q_{e}}\right)^{\frac{2}{3}}+\frac{2}{h^{2}}\left(\frac{4 \pi}{3}\right)^{\frac{2}{3}} U .
$$

Now, as we are going to apply (II) to the case where not only the atomic nuclei of various elements with their dissociated protons and electrons, but also the dissociated extranuclear electrons are present, $c_{P}$ and $c_{e}$ must be considered to contain much more than the protons and the electrons belonging to the element $E$.

At present, $U$ cannot be found from other than the mass-defect $\left(=\mu m_{P}-m_{k}\right)$, which is found from Aston's measurements of atomic weights.

$$
U=\left(\mu m_{P}-m_{E}\right) c^{2}
$$

where $\mu$ is taken as equal to the mass-number of an element." The result of the calculation is shown in Fig. 1. $U$ increases monotonously (almost linearly) with the mass-number of the element.

Though Aston's measurement does not yet extend to the element of the mass-number more than 200 , the mass-

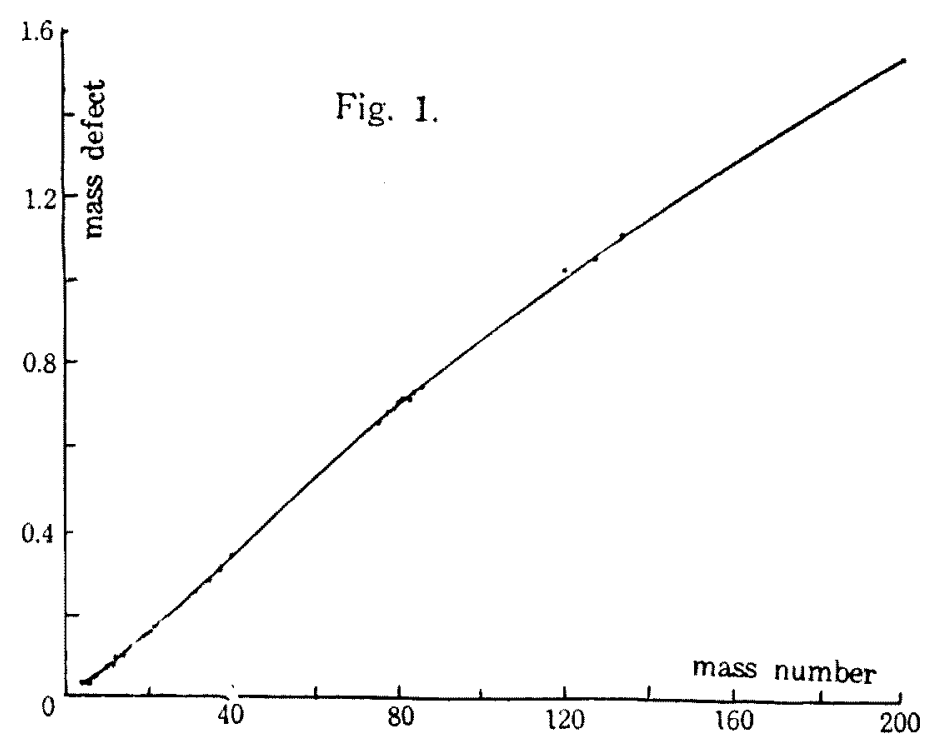
defect of heavy elements is easily estimated by a formula, which is

$$
\begin{aligned}
\text { mass-defect }= & \text { the mass-number of an element } \\
& \times \text { the packing fraction of a hydrogen atom, }
\end{aligned}
$$

because the packing fraction of a heavy element is small if compared with hydrogen. Thus, the heat of formation of heavy element increases in proportion to its mass-number.

1) E. A. Milne: Monthly Not., 90 (1930), 767.

2) $q_{e}$ is 2 as the spin of the electron is considered, and $q_{F}, q_{P}$ are possibly 1 .

3) $m_{P}$ and $m_{F}$ in (III) are obtained from Aston's measurement, in which the atomic weight of oxygen is taken as 16 . 
If all atomic nuclei in a white dwarf are really in the degenerate state, (II) teaches us that the nuclear concentration of an element increases enormously in proportion nearly to the cube of the massnumber, because not only $U$ but also $\mu$, $\nu$ are nearly proportional to the mass-number and all terms of the right hand in (II) are positive. This leads to an interesting conclusion that the heavy radio-elements are abundantly found in the interior of the white dwarf.

This conclusion is, however, justified if the concentration of electrons $c_{c}$ is really the least among those of all other elements. ${ }^{11}$ This is not true when all the dissociated extranuclear electrons are reckoned with, because the number of those electrons per atom increases with the atomic number. Therefore, as far as (II) remains valid and all particles are degenerate, it must be concluded that there is a "electrondeficit" in the interior of the star. Thus, a white dwarf is like an atom-model of Bohr, the highly dense star-core being positively charged and surrounded by the swarm of moving electrons.

This assumption may however be substituted by another to the effect that the star-interior is composed entirely of protons and electrons because, in such a way, (II) can be abandoned. Nevertheless, if a white dwarf is regarded as an enormously heavy atom, the first supposition is more plausible than anyone else.

If we assume as Milne did that the central region of the sun is degenerate, her atmosphere must be negatively charged.

The above consequences suggest us something about the structure of the ordinary atomic nucleus. Its internal state resembles much to the degenerated one, and the majority of the lodging protons and electrons does not remain isolated, but forms several groups in the nucleus, each of which is tightly bound and makes up a small nucleus, the heavy ones predominating. The extranuclear electrons are considered to appear as the result of expulsion from the nucleus due to Pauli's exclusion-principle. It may often happen that the distribution of the small nuclei in the mother nuclei is not in accordance with that derived from such an equilibrium equation as (II), in which case the superfluous particles (electrons, protons, alpha-particles etc.) may be ejected if bombarded by alpha-particles. This is the interpretation of the artificial disintegration of elements by Rutherford, Chadwick and others.

1) From (II), it is clearly seen that $c_{e}$ is very small if compared with $c_{E}$, as $m_{c} \ll m_{R}$. 
Appendix. The following thermal equilibrium equation of the dissociated atomic nuclei are produced here for reference.

In the case when both protons and electrons are degenerate,

$$
\begin{aligned}
\log \left(\frac{c_{F}}{q_{F}}\right)= & \frac{\mu h^{2} c_{P}^{\frac{2}{3}}}{\left(\frac{4 \pi}{3} q_{P}\right)^{\frac{2}{3}}\left(2 m_{P} k T\right)}+\frac{\nu h^{2} c_{e}^{\frac{3}{3}}}{\left(\frac{4 \pi}{3} q_{e}\right)^{\frac{2}{3}}\left(2 m_{e} k T\right)}+\frac{U}{k T} \\
& +3 \log \frac{\left(2 \pi m_{H} k T\right)^{\frac{1}{2}}}{h} .
\end{aligned}
$$

In the case when electrons alone are degenerate,

$$
\begin{aligned}
\log \frac{q_{P}^{\mu} c_{F}}{q_{F} c_{P}^{u}}= & \frac{\nu h^{2} c^{\frac{2}{3}}}{\left(\frac{4 \pi}{3} q_{e}\right)^{\frac{3}{3}}\left(2 m_{e} k T\right)}+\frac{U}{k T}-\frac{3}{2}(\mu-1) \log (2 k \pi T) \\
& -\log \left\{\left(\frac{m_{P}^{\mu}}{m_{E}}\right)^{\frac{2}{3}} \cdot \frac{1}{h^{3(\mu-1)}}\right\} .
\end{aligned}
$$

In the case when all constituents are none-degenerate (Saha's formula),

$$
\begin{aligned}
\log \frac{q_{P}^{\mu} q_{e}^{\nu} c_{R}}{q_{F} c_{P}^{\nu} c_{e}^{\nu}}= & \frac{U}{k T}-\frac{3}{2}(\mu+\nu-1) \log (2 \pi k T) \\
& -\log \left\{\left(\frac{m_{P}^{\mu} m_{e}^{\nu}}{m_{F}}\right)^{\frac{2}{3}} \frac{1}{h^{3(\mu+\nu-1)}}\right\} .
\end{aligned}
$$

